\title{
Front-end Solar Temperature And Humidity Control System
}

\author{
Haocheng. Dai ${ }^{1^{*}}$ \\ ${ }^{1}$ Department of energy and power engineering, Wuhan University of Technology, Wuhan, Hubei, 430000, China
}

\begin{abstract}
Solid dehumidification is one of the important components of the dehumidification airconditioning system. As an important method of solid dehumidification, the dehumidification performance and regeneration performance of fixed-bed dehumidification have an important impact on the operation and energy saving of the dehumidification air-conditioning system. In view of the high air temperature and high humidity in South China, our design organically combines dehumidification fixed beds, semiconductor refrigeration devices, foam ceramic insulation panels and double-glazed windows. We have designed a photovoltaic-driven modular solar temperature regulating and dehumidifying purification wall suitable for building dehumidification. The dehumidification window can use daytime solar radiation to regenerate the dehumidification fixed bed after absorbing moisture, reducing the energy consumption of regeneration. At the same time, the design can also use semiconductor refrigeration devices to adjust the temperature and humidity of the window inlet air to improve the window's dehumidification and regeneration performance. We tested and studied the dehumidification and regeneration performance of the device under different working conditions through experimental tests, which provided a basis for the engineering application and theoretical analysis of the device.
\end{abstract}

\section{Introduction}

Hot and humid weather not only affects people's comfort, but also brings many inconveniences to people's life and work, and increases air conditioning energy consumption. Therefore, dehumidifying the air can not only improve the quality of people's working and living environment, but also reduce the energy consumption of the air conditioning system, which is in line with the current national development theme of energy conservation and emission reduction [1]. Commonly used air dehumidification methods are: cooling dehumidification method, compression dehumidification method, solution absorption dehumidification method and solid adsorption dehumidification method, or a dehumidification method combining the above methods. At the same time, some scholars have proposed some new dehumidification technologies, such as: membrane dehumidification, electrochemical dehumidification and heat pump dehumidification [2]. Among them, solid adsorption dehumidification has the advantages of large processing air volume, low-grade energy sources for regeneration, easy realization of internal cooling dehumidification and internal heat regeneration, simple structure and maintenance, low noise and reliable operation.

After the fixed bed is dehumidified and saturated, it needs to be regenerated to realize cyclic operation. The regeneration performance of the fixed bed is an important factor affecting the dehumidification performance of the fixed bed. The traditional method of fixed bed regeneration is electric heating regeneration [3]. The principle is to use electric energy to directly heat air to regenerate the fixed bed. However, this method has the main problems of low regeneration efficiency, high energy consumption, and long time [4]. In response to this, many scholars have proposed new regeneration methods, including solar regeneration, waste heat and waste heat regeneration, ultrasonic regeneration, electroosmosis regeneration, and microwave regeneration. Among them, solar energy regeneration has a good energy-saving effect, can effectively alleviate the environmental pollution caused by the burning of fossil fuels, and will not cause harm to the human body [5]. At the same time, the annual accumulated sunshine hours in South China range from 1,200 to 2,200 hours, and the solar radiation range is about 4086.6 to $5225.1 \mathrm{MJ} / \mathrm{m} 2$. It has abundant solar energy, which provides a good energy environment for solar regeneration fixed beds [6]. At present, the research and development of solar energy regeneration fixed beds are relatively mature abroad, and there are few related researches in China.

In view of the advantages of fixed beds in air dehumidification, problems in regeneration and the thermoelectric effect of semiconductors, this paper combines fixed dehumidifying beds, semiconductor refrigeration devices and solar photovoltaic panels to develop a solar energy conditioning system suitable for civil buildings [7]. At the same time, it has the characteristics of not occupying building space, heat preservation and heat insulation, and realizes energy saving in many aspects [8].

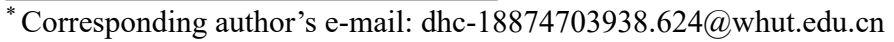




\section{Materials and Methods}

\subsection{Working principle}

2.1.1 Solar energy utilization process. When the equipment is running, the photovoltaic panels on the roof and window eaves use the photovoltaic effect of the solar cell semiconductor materials to convert solar energy into electrical energy, and the electrical energy is collected and stored in the battery. The inverter converts direct current into alternating current, and provides $220 \mathrm{~V}$ voltage to the semiconductor cooling fins, heat pipe radiators and selfhanging louver axial exhaust fans in the temperature control module. On the other hand, the regeneration of the dehumidifying layer can directly use solar radiation to regenerate the solid dehumidifying material.

\subsubsection{Temperature adjustment and dehumidification} process. The two fans inside the window work (the fans on both sides of the window are not working at this time), and the outdoor high-humidity air is driven by the fan to enter the temperature and humidity control module from the air inlet. In the temperature and humidity control module, the air in the upper channel is heated by the hot end of the semiconductor device and discharged to the outside, and the air in the lower channel is cooled by the cold end of the semiconductor device and sent into the window. The cooled air passes through the dehumidification layer in the window to reduce the air humidity, and then is sent into the room through the fan.

2.1.3 Regeneration process. The regeneration of the dehumidification layer directly uses solar radiation to regenerate the solid dehumidification material. Sunlight irradiates the dehumidification layer, the solid dehumidification material in the dehumidification layer absorbs solar radiation and the temperature rises, and the moisture in the solid dehumidification material is heated and converted into water vapor to be released. At this time, the fans on both sides of the window work, and the outdoor air is driven by the fans to enter the temperature and humidity control module from the air inlet. In the temperature and humidity control module, the air in the lower channel is cooled by the cold end of the semiconductor device and discharged to the outdoors, and the air in the upper channel is heated by the hot end of the semiconductor device and sent into the window. The heated air enters the window to further heat the solid dehumidifying material, and discharge the water vapor released by the dehumidifying material from the fans on both sides of the dehumidifying window.

\subsection{Device design}

The solar energy temperature control and dehumidification purification wall experimental test device we designed is mainly composed of five parts: air treatment section, temperature and humidity control module, dehumidification window, solar radiation simulation system and simulation chamber.

2.2.1 Air treatment section. The air treatment section includes wooden air ducts, PTC heaters and ultrasonic humidifiers. The wooden air duct is divided into an inlet section and a mixing section. The inlet section is equipped with adjustable power DJR heaters and ultrasonic humidifiers to provide the temperature and humidity conditions of the inlet air under different working conditions. The processed air is fully in the mixing section. mixing.

2.2.2 Temperature and humidity control module. The main structure of the temperature and humidity control module is mainly composed of a $60 \mathrm{~mm}$ thick foam ceramic board and a $20 \mathrm{~mm}$ thick extruded board, with a length $\times$ width $\times$ height of $1100 \times 240 \times 390 \mathrm{~mm}$. The upper and lower passages of the module are separated by $4 \mathrm{~mm}$ thick wooden boards and covered with $20 \mathrm{~mm}$ thick extruded boards. The cross-sectional dimensions of the upper and lower passages are both $120 \times 110 \mathrm{~mm}$. There are 5 semiconductor refrigeration fins with a power of $60 \mathrm{~W}$ in the wood board, the refrigeration fins are connected in parallel, the upper end of the refrigeration fin is the hot end, the lower end is the cold end, and the two ends are respectively connected to the heat pipe radiator.

\subsubsection{Design of dehumidification window. The main} structure of the dehumidification window is mainly composed of $15 \mathrm{~mm}$ thick wooden boards and $3 \mathrm{~mm}$ ordinary glass, with a length $\times$ width $\times$ height of $1150 \times$ $200 \times 1600 \mathrm{~mm}$, and the outer wooden structure is covered with $15 \mathrm{~mm}$ thick insulation materials. The structure of the dehumidification window includes three parts: a dehumidification layer, an air inlet and an air outlet. The window spacing between double-glazed windows is $200 \mathrm{~mm}$, the outside glass is fixed, and the inside is made of a movable door for the convenience of material replacement. The air inlet is located on the lower side of the outer window; 3 dehumidifying layers are set in the window. The dehumidification layer is composed of 6 movable dehumidification modules of the same size $(550 \times 190 \times 50 \mathrm{~mm})$, and the thickness of the dehumidification material is $50 \mathrm{~mm}$. The air supply port is located on the upper part of the inner side of the window. At the air supply port, 2 sets of self-hanging louvered axial exhaust fans are set to send air into the room. The air outlet is set on the outside of the upper part of the window, and 2 fans are installed to eliminate the regeneration of the dehumidification layer. Hot and humid air at times.

2.2.4 Solar radiation simulation system. The solar radiation simulation system includes a solar radiation simulator, a solar pyrometer and a solar radiation observation station. In this experiment, 4 sets of $2000 \mathrm{~W}$ solar radiation simulators are used to simulate the solar radiation intensity under different working conditions. 
2.2.5 Simulation room. The simulation room is composed of a $50 \mathrm{~mm}$ thick color steel sandwich panel with a length $\times$ width $\times$ height of $1250 \times 1200 \times 2000 \mathrm{~mm}$, which is used to test the changes in temperature and humidity of the exhaust air of the solar dehumidification window

\section{Results and Discussion}

In this paper, the dehumidification, regeneration and temperature control performance test of the developed modular solar temperature and humidity control wall is carried out. The experimental test period is summer in Guangzhou, and its temperature and relative humidity are the highest periods in the region. The test parameters collect a set of data every 5 minutes. Because the temperature and humidity of the inlet and outlet are constantly fluctuating, the average temperature and humidity of the 12 sets of data recorded every hour is taken as the calculated value of temperature and humidity in the period. Then the calculated value of temperature and humidity in the hourly period is converted into air humidity, and the air humidity is directly used to analyse the dehumidification regeneration performance.

\subsection{Layout of measuring points and test instruments}

We use color steel sandwich panels to build a simulation device with a length $\times$ width $\times$ height of $1200 \mathrm{~mm} \times$ $200 \mathrm{~mm} \times 2000 \mathrm{~mm}$. The experimental locations include: temperature and humidity probes are evenly distributed on the inlet and outlet of the temperature and humidity purification wall, the dehumidification layer and the outdoor; the six solid dehumidification modules are evenly distributed with three upper, middle and lower thermocouples; the surface of the inner window glass is evenly arranged 4 thermocouples and 2 heat flux plates; thermocouples are arranged in the indoor space, inner wall,

and outer wall for temperature measurement.

\subsection{Effective dehumidification time}

The solid dehumidifier is dried in a drying box at $120^{\circ} \mathrm{C}$, and its mass is weighed, and then placed in a temperature and humidity control purification wall. Run the temperature and humidity purification wall, when the high-temperature and high-humidity air flows through the window, measure the temperature and humidity of the inlet and outlet air; when the temperature and humidity of the outlet air does not change much or remain unchanged, this is the end of the dehumidification operation. The time is the theoretical maximum dehumidification time of the temperature regulation and dehumidification purification wall. Also called theoretical effective dehumidification time. In this paper, because the air passes through the dehumidification purification window for a long time, the dehumidification efficiency is in a rapid growth process at the beginning, so this paper defines the actual effective dehumidification time from the moment when the maximum dehumidification capacity is initially reached to the moment when the dehumidification capacity drops to 0 .

The parameters of the four test conditions are shown in Table 1. The hourly dehumidification volume change curve is a curve that first rises quickly and then drops slowly. The effective dehumidification time periods under the four working conditions are: $2 \sim 28 \mathrm{~h}, 2 \sim 27 \mathrm{~h}, 2 \sim 35 \mathrm{~h}$ and $2 \sim 27 \mathrm{~h}$. The actual effective dehumidification time is $27 \mathrm{~h}$, $26 \mathrm{~h}, 34 \mathrm{~h}$ and $26 \mathrm{~h}$ respectively; the average effective dehumidification time is $28.25 \mathrm{~h}$. It shows that the modular solar temperature control and dehumidification purification wall has dehumidification function within 26 hours, which is much higher than the effective dehumidification time of current solid dehumidifiers, and can reduce the air humidity entering the room for a long time.

Table 1. Imported parameters under four test conditions.

\begin{tabular}{cccc}
\hline $\begin{array}{c}\text { Dehumidification } \\
\text { conditions }\end{array}$ & $\begin{array}{c}\text { Imported average dry bulb } \\
\text { temperature }\left({ }^{\circ} \mathrm{C}\right)\end{array}$ & $\begin{array}{c}\text { Average relative humidity of } \\
\text { imports }(\%)\end{array}$ & $\begin{array}{c}\text { Imported moisture } \\
\text { content }(\mathrm{G} / \mathrm{kg})\end{array}$ \\
\hline Working condition 1 & 27.1 & 63.6 & 14.33 \\
Working condition 2 & 26.7 & 64.2 & 14.13 \\
Working condition3 & 24.7 & 65.2 & 12.71 \\
Working condition 4 & 26.6 & 84.7 & 18.66 \\
\hline
\end{tabular}

\subsection{Dehumidification efficiency}

Dehumidification efficiency is one of the important indicators reflecting the dehumidification performance of the temperature-regulating dehumidification purification wall, which is defined as the ratio of the dehumidification amount to the moisture content of the inlet air. The calculation formula is

$$
\eta_{1}=\frac{W_{1}-W_{2}}{W_{1}} \times 100 \%
$$

The hourly dehumidification efficiency changes under the four working conditions are shown in Table 2. It can be seen that the change trend of the hourly dehumidification efficiency change curve and the hourly dehumidification capacity change curve are basically the same. Since the effective dehumidification time of this device is relatively long, and the hourly dehumidification efficiency change curve gradually decreases during the effective dehumidification time, the research and analysis are carried out in sections.

It can be seen from the above results that:

a) The average dehumidification efficiency of the temperature-adjusting and dehumidifying purification wall in each time period of $1 \sim 5 \mathrm{~h}$, 
$6 \sim 10 \mathrm{~h}, 11 \sim 15 \mathrm{~h}, 16 \sim 20 \mathrm{~h}$ and $21 \sim 25 \mathrm{~h}$ is $14.11 \%$, $10.04 \%, 7.05$, respectively $\%, 5.02 \%$ and $2.92 \%$.

b) The average dehumidification efficiency curve shows a significant downward trend with the test period.

Table 2. Average dehumidification efficiency of each time period of dehumidification curve (\%)

\begin{tabular}{cccccc}
\hline & $1 \sim 5 \mathrm{~h}$ & $6 \sim 10 \mathrm{~h}$ & $11 \sim 15 \mathrm{~h}$ & $16 \sim 20 \mathrm{~h}$ & $21 \sim 25 \mathrm{~h}$ \\
\hline Working condition 1 & 15.35 & 12.06 & 8.43 & 4.70 & 2.13 \\
Working condition 1 & 13.14 & 8.95 & 5.67 & 3.40 & 1.74 \\
Working condition 1 & 20.81 & 14.54 & 10.18 & 7.31 & 5.53 \\
Working condition 1 & 7.12 & 4.60 & 3.92 & 4.68 & 2.26 \\
Average division & 14.11 & 10.04 & 7.05 & 5.02 & 2.92 \\
Wet efficiency & & & \\
\hline
\end{tabular}

\subsection{Inlet and outlet air temperature rise}

When the solid dehumidifier is in the dehumidifying state, the moisture in the air is converted from gas to liquid and will release latent heat of vaporization. The heat released will increase the air temperature and increase the air conditioning load. The latent heat released by the dehumidification window during the dehumidification and outlet air. In the four test conditions, the inlet and outlet air temperature rises were $3.86^{\circ} \mathrm{C}, 3.50^{\circ} \mathrm{C}, 6.92^{\circ} \mathrm{C}$ and $3.30^{\circ} \mathrm{C}$, and the average inlet and outlet temperature rise was $4.38^{\circ} \mathrm{C}$. The test results show that the temperature rise of the inlet and outlet air is directly proportional to the dehumidification efficiency, that is, the higher the dehumidification efficiency, the greater the temperature rise, reflecting the greater the latent heat released.

\subsection{Regeneration performance}

We use color steel sandwich panels to build a simulation device with a length $\times$ width $\times$ height of $1200 \mathrm{~mm} \times$ $200 \mathrm{~mm} \times 2000 \mathrm{~mm}$. The experimental locations include: temperature and humidity probes are evenly distributed on the inlet and outlet of the temperature and humidity purification wall, the dehumidification layer and the outdoor; the six solid dehumidification modules are evenly distributed with three upper, middle and lower thermocouples; the surface of the inner window glass is thermocouples are arranged in the indoor space, inner wall, and outer wall for temperature measurement.

The calculation results of regeneration efficiency test under different working conditions show that under four different radiation intensities of 1200, 900, 600 and $300 \mathrm{~W} / \mathrm{m} 2$ :

a) The average regeneration efficiency of pure radiation regeneration is $20.06 \%, 18.04 \%, 16.32 \%$ and $13.15 \%$ respectively.

b) The average regeneration efficiency of the radiation + natural ventilation regeneration method is $14.63 \%, 11.54 \%, 9.11 \%$ and $8.34 \%$ respectively.

c) The regeneration efficiency of the radiation + air conditioning exhaust regeneration method is relatively low, being $8.80 \%, 8.04 \%, 5.36 \%$ and $1.91 \%$ respectively. time can be reflected by the temperature rise of the inlet evenly arranged 4 thermocouples and 2 heat flux plates; c) The moisture content of the inlet air has a greater impact on the dehumidification efficiency. The greater the moisture content, the dehumidification efficiency The smaller. d) The average regeneration efficiency of radiation + rotary dehumidification regeneration method is $15.92 \%, 13.51 \%, 11.93 \%$ and $8.80 \%$ respectively.

Its research shows that: the regeneration efficiency of the dehumidification purification window is proportional to the radiation intensity; the pure radiation regeneration efficiency is higher than the regeneration efficiency of other combinations under the same radiation intensity; at the same time, the temperature and humidity changes of the air inlet and the environment have a greater impact on the regeneration efficiency.

\section{Conclusions}

The new modular solar temperature and humidity control wall designed by us organically combines solar photovoltaic panels, semiconductor refrigeration sheets, double-glazed windows and foam ceramic heat insulation panels, which expands the functions of pure foam ceramic heat insulation and semiconductor refrigeration. The integration of systems and buildings and the development of prefabricated buildings provide a feasible engineering application mode.

The device and system developed are not reported in the literature at home and abroad. The design idea is reasonable, the structure is unique, and the principle of non-power recycling is ingenious and feasible. The system has been tested in the laboratory and can be applied to most non-structural walls in houses. It has good engineering feasibility and applicability, and solves the heat and humidity problems of residents to a certain extent. It is suitable for popularization and application in areas with high humidity.

\section{References}

1. Jiang Yi, Yang Xiu. my country's building energy consumption status and problems in building energy efficiency[J]. China Construction, 2006(2): 12-18.

2. Wei Qingpeng, Wang Xin, Xiao He, et al. Current status and characteristics of energy consumption in public buildings in China[J]. Building Science and Technology, 2009(8): 38-40.

3. Li Nan, Liao Jianke, Zheng Wenqian, Liu Hong. Thermal comfort analysis of a combined air conditioning system with cooling ceiling and 
displacement ventilation[J]. Journal of Central South University (Natural Science Edition), 2012, 43(06): 2434-2440.

4. Shenzhen Academy of Building Research Co., Ltd. Green Office Building Design and Cases in South China [M]. Shenzhen: China Construction Industry Press, 2016.

5. Yeboah S K, Darkwa J. A critical review of thermal enhancement of packed beds for water vapour adsorption $[\mathrm{J}]$. Renewable and Sustainable Energy Reviews, 2016, 58: 1500-1520.

6. Misha S, Mat S, Ruslan M H, et al. Review of solid/liquid desiccant in the drying applications and its regeneration methods $[\mathrm{J}]$. Renewable and sustainable energy reviews, 2012, 16(7): 4686-4707

7. Hamed A M. Desorption characteristics of desiccant bed for solar dehumidification/humidification air conditioning systems [J]. Renewable Energy, 2003, 28(13): 2099-2111.

8. Yadav A, Bajpai V K. Experimental comparison of various solid desiccants for regeneration by evacuated solar air collector and air dehumidification [J]. Drying Technology, 2012, 30(5): 516-525. 